\title{
Kinetic Modeling of Dopant and Impurity Surface Segregation During Vapor Phase Growth: Multiple Mechanism Approach
}

\section{Citation}

Arnold, Craig B. and Michael J. Aziz. 2003. Kinetic modeling of dopant and impurity surface segregation during vapor phase growth: Multiple mechanism approach. Materials Research Society Symposia Proceedings 749: W14.3.1-W14.3.7.

\section{Published Version}

http://www.mrs.org/s_mrs/sec_subscribe.asp?CID=2572\&DID=118880\&action=detail

\section{Permanent link}

http://nrs.harvard.edu/urn-3:HUL.InstRepos:2795432

\section{Terms of Use}

This article was downloaded from Harvard University's DASH repository, and is made available under the terms and conditions applicable to Other Posted Material, as set forth at http:// nrs.harvard.edu/urn-3:HUL.InstRepos:dash.current.terms-of-use\#LAA

\section{Share Your Story}

The Harvard community has made this article openly available.

Please share how this access benefits you. Submit a story.

\section{Accessibility}




\title{
KINETIC MODELING OF DOPANT AND IMPURITY SURFACE SEGREGATION DURING VAPOR PHASE GROWTH: MULTIPLE MECHANISM APPROACH
}

\author{
Craig B. Arnold ${ }^{1}$ and Michael J. Aziz ${ }^{2}$ \\ ${ }^{1}$ Materials Science and Technology Division \\ Naval Research Laboratory, Washington, DC 20375, USA \\ ${ }^{2}$ Division of Engineering and Applied Sciences, \\ Harvard University, Cambridge, MA 02138, USA
}

\begin{abstract}
We propose a new kinetic model for surface segregation during vapor phase growth that accounts for multiple segregation mechanisms, including mechanisms for terrace mediated exchange and step edge mediated exchange. The major result of the model is an analytic expression for the experimentally measured segregation length and profile broadening that can be readily calculated without the need for numerical simulations. We compare the model to experimental measurements for the temperature dependence of segregation of $\mathrm{Sb}$ in $\mathrm{Si}(001)$. The model is able to accurately describe both the anomalous segregation at low temperature and the transition between equilibrium and kinetically limited segregation at high temperature. An excellent agreement is obtained using realistic energies and pre-exponential factors for the kinetic rate constants. The model can be applied to other segregating systems in planar geometries, including metallic and III-V semiconducting thin films.
\end{abstract}

\section{INTRODUCTION}

Sharp interface structures have become increasingly important to advanced devices. Two relevant examples include semiconductor delta doping for quantum well devices $[1,2]$ and multilayered metallic systems for magnetic storage devices [3,4]. The basic challenge in these cases is to overcome the classical problem of segregation whereby one atomic species tends to segregate to the free surface during deposition. For these advanced devices, segregation affects not only the quality of a device but also its ability to function at all. The phenomenon is general to almost any system including complex semiconductors, metals and insulators, although it has been studied most quantitatively in attempts to attain sharp doping profiles in silicon [5].

There had been extensive work on understanding the problem of segregation almost two decades ago when simple kinetic models explained important features of the early experimental results. These models predicted experimentally observed transitions from local equilibrium to kinetically trapped segregation regimes as with decreasing temperature and indicated that the only way to overcome segregation is to go to lower growth temperatures where, unfortunately, epitaxial growth tends to break down. However, it was not until the 1990's that low-temperature epitaxial growth techniques were developed and enabled this low temperature segregation regime to be experimentally studied [6]. It was found that the previous models could not accurately describe the observed behavior at lower temperatures.

Our objective is to reexamine the kinetics of segregation in light of these low temperature segregation regimes and to develop a model that successfully explains the observed behavior. To 
this end, we have incorporated multiple mechanisms for segregation, including step-edge and terrace mediated processes. Our model results compare favorably to experimentally observed behavior for $\mathrm{Sb}$ in $\mathrm{Si}(001)$ using realistic barrier energies and prefactors. Through this model, we are able to attribute the anomalous low-temperature segregation behavior to step-edge mediated processes.

\section{PREVIOUS MODELS}

The previous kinetic models for the segregation of dopants in semiconductor systems can be classified into two categories based on the location of the segregation or incorporation events. The first category, atomic exchange events occur at terraces on the surface $[7,8,9, ?]$. In this case, the surface is treated as perfectly flat with no step edges and deposition occurs in a layer-by-layer fashion at a single moment. Atoms are allowed to exchange places between the surface layer and subsurface layers until the next layer is deposited and the atoms in the subsurface layer are trapped in the film. This basic model with minor modifications was very successful at describing the observed behavior for segregation in a wide variety of semiconductor systems including III-V's and silicon. These models predict a transition between a kinetically limited segregation regime in which the solute atoms do not have enough mobility to segregate out of the growing film before the next layer is deposited, and an equilibrium regime in which the atoms have enough energy to surmount the energy barrier for exchange and the segregation behavior is dictated by local thermodynamic equilibrium in the system. The major problem with these models is that they fail to account for the amount of segregation that occurs at low temperatures.

In response to the shortcomings of the terrace exchange models, models were developed based on exchange events occurring primarily at the step edges $[11,12]$. The behavior at lower temperatures was well described by these models, but they did not predict the transition to equilibrium segregation at the higher temperatures.

\section{NEW MODEL}

Our new model for surface segregation combines both terrace and step-edge mediated exchange processes and applies the steady-state approach successfully developed for liquid-solid segregation $[7,13]$. The first step is to simplify the surface by considering a 2-dimensional structure of periodic steps on the surface with implicit uniformity in the direction into the plane of the page (Fig. 1). The stepped surface is separated into step-edge regions (S,E), terrace regions $(\mathrm{T}, \mathrm{P})$ and adatom regions $(\mathrm{M}, \mathrm{R})$. By simplifying the surface in this manner, we account for all the specific transitions in a broad way by considering only direct interchanges between the neighboring regions. We are not concerned with all of the detailed kinetic pathways - these may involve point defects which would involve too much detail to account for. Rather, the direct interchange pathway in the model reflects an effective statistical average of the various actual kinetic pathways in the actual system, which is presumably dominated by the pathway with the lowest barrier in most situations.

Our reference frame is centered on the moving step edge at $x=0$. The rate of change of the concentration of impurity atoms in each of the six regions is determined by tracking the mass into and out of the regions. Mass balances consist of up to four terms; 1)vertical diffusion into and out 


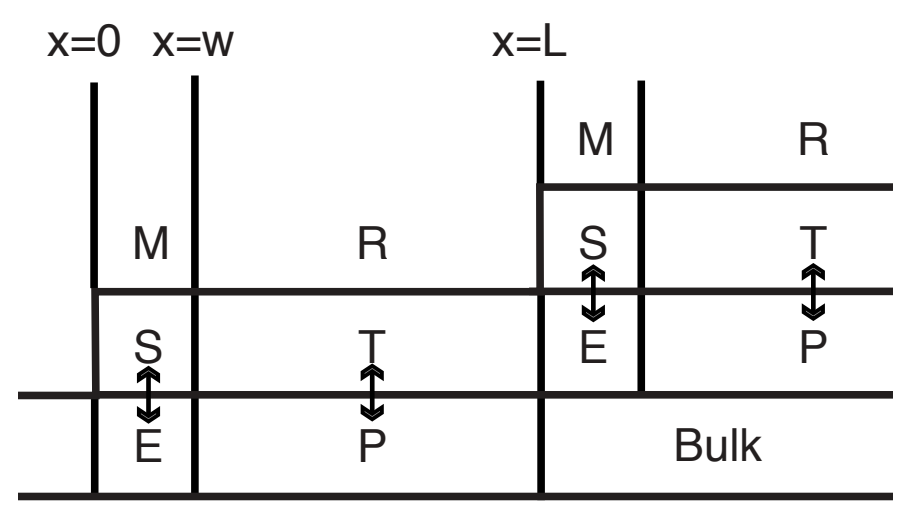

Figure 1. Simplification of surface processes

of the region, 2)lateral diffusion within a layer, 3) convective flux due to the fact that the layer boundaries are moving in the $\mathrm{x}$ direction with respect to the lattice, and 4) deposition and evaporation flux in the topmost layers. For example, the terrace region $\mathrm{T}$ has material entering and leaving through regions $\mathrm{R}$ and $\mathrm{P}$ by diffusive transport. It also has lateral diffusion smearing out the profile as well as convective flux entering from region $\mathrm{S}$ and leaving through region $\mathrm{E}$.

A mass balance performed this way on each of the six regions produces a set of six coupled second order partial differential equations that is solvable with the appropriate boundary conditions. However, this approach requires numerical approximations and complicated solutions that obscure the physical interpretation of the kinetic model; it also makes it difficult to compare our results to real experiments where the relevant energies are unknown at this time. With the following set of simplifying assumptions we reduce the problem to one that is readily solved and implemented for comparison to experimental conditions.

The first and most significant assumption is that we consider only two segregation mechanisms: one terrace-mediated mechanism and one step edge-mediated mechanism. These are sufficient to account for the relative importance of the two different types of mechanisms. For this development, we have chosen to keep the S-E and T-P transitions. Given space limitations we assert the following three points that can be shown to be valid with reasonable assumptions. First, diffusion within a layer does not contribute significantly to the overall segregation behavior. Certainly diffusion will play a role in determining the actual lateral composition profiles in the given regions, but the calculation of the experimentally observable segregation parameter requires only the integrated amount of solute in a layer and not its distribution within the layer. Next, we allow the step edge region to approach the dimensions of a single lattice spacing. This allows us to treat the step edge region as discrete and remove any spatial derivatives. Finally, we assume that the adatom regions $\mathrm{M}$ and $\mathrm{R}$ do not contribute significantly to the overall Gibbsian surface excess of impurity atoms, which is dominated by those in the other non-bulk regions.

These assumptions result in a set of 4 coupled first order partial differential equations:

$$
\left.\frac{\partial C^{P}(x, t)}{\partial t}=\frac{1}{a}\left(J^{T P}-J^{P T}\right)\right)-v \frac{\partial C^{P}(x, t)}{\partial x}
$$




$$
\begin{aligned}
\frac{d C^{E}(t)}{d t} & =\frac{1}{a}\left(J^{S E}-J^{E S}\right)-\frac{v}{a_{o}} \cdot\left(C^{E}(t)-C^{T}(L, t)\right) \\
\frac{\partial C^{T}(x, t)}{\partial t} & \left.=\frac{1}{a}\left(J^{P T}-J^{T P}\right)\right)-v \frac{\partial C^{T}(x, t)}{\partial x} \\
\frac{d C^{S}(t)}{d t} & =\frac{1}{a}\left(J^{E S}-J^{S E}\right)-\frac{v}{a_{o}} \cdot\left(C^{S}(t)-C^{b u l k}\right) .
\end{aligned}
$$

In these equations, $C^{\alpha}$ represents the concentration of impurity atoms in region $\alpha$ (S,T,E,P,bulk), $J^{\alpha \beta}$ is the diffusive flux of impurity atoms from region $\alpha$ to $\beta, a$ is the interplanar spacing in the vertical direction, $a_{o}$ is the interatomic spacing within the plane, and $v$ is the velocity of the moving step edge. The $J^{\alpha \beta}$ term depends on the concentration in each region and is derived from standard transition rate theory for the reaction coordinate diagram shown in Fig. 2.Similar expressions are obtained for all the other inter-region diffusive transitions. In the dilute concentration limit the diffusive fluxes are of the form,

$$
\begin{aligned}
J^{P T} & =C^{P}(t) \cdot \nu a \cdot \exp \left(-\frac{Q^{T P}}{k_{B} T}\right) \\
J^{T P} & =C^{T}(t) \cdot \nu a \cdot \exp \left(-\frac{\left(Q^{T P}+\Delta \mu^{\prime T P}\right)}{k_{B} T}\right) .
\end{aligned}
$$

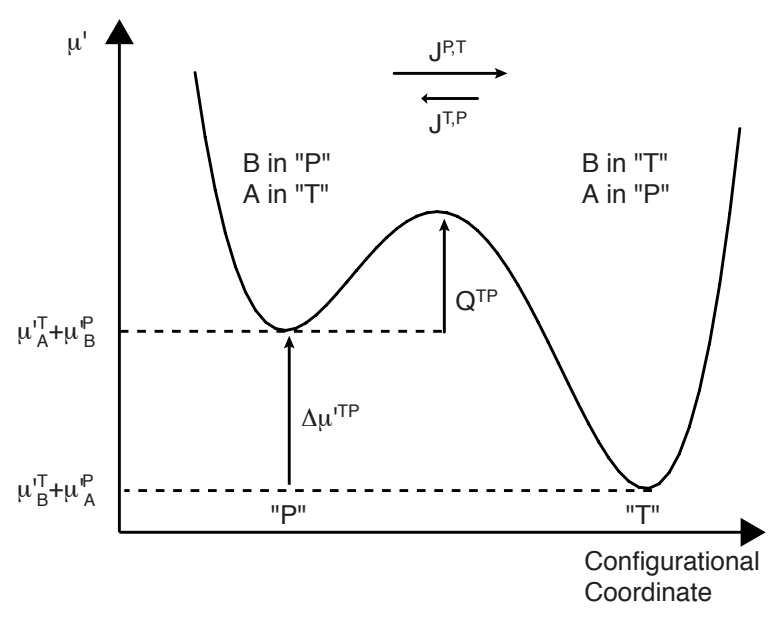

Figure 2. Energy diagram for the T-P transition. $\mu^{\prime}$ is chemical potential minus the contribution from the ideal entropy of mixing.

In order to solve this system of equations (1-4), we substitute the explicit equations for flux and go to the steady state regime. This enables us to equate all the time derivative to zero and directly solve a set of ordinary differential equations. The solution is

$$
\begin{aligned}
& C^{P}(x)=-\mathbb{A e}^{-\kappa x}+k_{e}^{T P} \mathbb{B} \\
& C^{T}(x)=\mathbb{A} \mathrm{e}^{-\kappa x}+\mathbb{B},
\end{aligned}
$$




$$
\begin{aligned}
C^{S} & =\mathbb{A e}^{-\kappa a_{o}}+\mathbb{B} \\
C^{E} & =-\mathbb{A e}^{-\kappa a_{o}}+k_{e}^{T P} \mathbb{B} .
\end{aligned}
$$

where

$$
\begin{aligned}
\kappa & =\frac{v_{d}^{T P}}{v a_{o}}\left(1+k_{e}^{T P}\right) \\
k_{e}^{\alpha \beta} & =\exp \left(\frac{-\Delta \mu^{\prime \alpha \beta}}{k_{B} T}\right) \\
v_{d}^{\alpha \beta} & =\nu a_{o} \exp \left(\frac{-Q^{\alpha \beta}}{k_{B} T}\right) .
\end{aligned}
$$

And,

$$
\begin{aligned}
& \mathbb{A}= C^{b u l k} \mathrm{e}^{\kappa a_{o}} \frac{k_{e}^{T P}\left(\frac{v}{v_{d}^{S E}}+1\right)-k_{e}^{S E}-\frac{v}{v_{d}^{S E}}}{\mathrm{e}^{-\kappa\left(L-a_{o}\right)}\left(\frac{v}{v_{d}^{S E}}+k_{e}^{S E}-k_{e}^{T P}\right)+k_{e}^{T P}\left(1+k_{e}^{S E}+\frac{v}{v_{d}^{S E}}\right)} \\
& \mathbb{B}=C^{b u l k} \frac{\frac{v}{v_{d}^{S E}}\left(1+e^{-\kappa\left(L-a_{o}\right)}\right)+1+k_{e}^{S E}}{\mathrm{e}^{-\kappa\left(L-a_{o}\right)}\left(\frac{v}{v_{d}^{S E}}+k_{e}^{S E}-k_{e}^{T P}\right)+k_{e}^{T P}\left(1+k_{e}^{S E}+\frac{v}{v_{d}^{S E}}\right)} .
\end{aligned}
$$

We are now in a position to predict an experimentally measurable quantity from these atomic fractions. There are a variety of measures used in the literature, but for convenience we use the segregation ratio $r$ as defined by Jorke [9]:

$$
r \equiv \frac{\text { Surface Areal Concentration of Impurity }}{\text { Bulk Volume Concentration }}
$$

Substituting, we obtain the relatively simple final result,

$$
r=a\left(1+k_{e}^{T P}\right) \frac{\mathbb{B}}{C^{b u l k}} .
$$

The above equation represents the main result of this model. Looking at equation 15, we can see the main physical/experimental dependencies in our model. Most importantly, the parameters depend on the temperature through terms such as $k_{e}^{S E}, v_{d}^{S E}$, and $\kappa$. But also there is a deposition rate dependence through $v$ as well as a terrace length or surface orientation or morphology dependence.

\section{COMPARISON TO EXPERIMENTS}

When we compare the result of equation 17 , we are able to reproduce the experimentally measured segregation ratio for $\mathrm{Sb}$ in $\mathrm{Si}(001)$ in all temperature regimes(Fig. 3) using the 
parameters given in Table I . The fit from equation 17 is shown by a solid line, while dashed and dotted lines show the fits for our model if we consider only the terrace-mediated or step edge-mediated process, respectively. We interpret the "anomalous" low temperature behavior as the result of a step edge mechanism working in parallel with the terrace mechanism but with a lower activation barrier. This is consistent with our expectation that a step edge atom must break fewer bonds to move than does a terrace atom. Furthermore, the smaller fitted value of $\Delta \mu^{\prime} S E$ compared to $\Delta \mu^{\prime T P}$ indicates that the the solute atom sees less of a difference between the two states at a step edge than in mid-terrace.

A necessary consequence of incorporating two mechanisms in the model is the inclusion of four relevant energies which results in four fitting parameters. Each mechanism has an activation barrier and a potential difference associated with it. However, by looking at other experimental dependencies such as the deposition flux dependence, we can effectively reduce the number of free parameters by further restricting the segregation behavior.

Table I. Table of the parameters used in our model to fit the data in Fig. 3. The first column represents variables used as fitting parameters while the second column represents those used as fixed parameters.

\begin{tabular}{|c|c||c|c|}
\hline Fitting parameters & & Fixed Parameters & \\
\hline$Q^{T P}$ & $1.90 \mathrm{eV}$ & $\nu$ & $1 \times 10^{13} \mathrm{~s}^{-1}$ \\
$\Delta \mu^{\prime T P}$ & $1.03 \mathrm{eV}$ & $a_{o}$ & $5.432 \times 10^{-8} \mathrm{~cm}$ \\
$Q^{S E}$ & $1.19 \mathrm{eV}$ & $a$ & $1.358 \times 10^{-8} \mathrm{~cm}$ \\
$\Delta \mu^{\prime S E}$ & $0.37 \mathrm{eV}$ & $L$ & $25 \cdot a_{o}$ \\
& & $R$ & $1 \mathrm{~mL} \cdot \mathrm{s}^{-1}$ \\
\hline
\end{tabular}

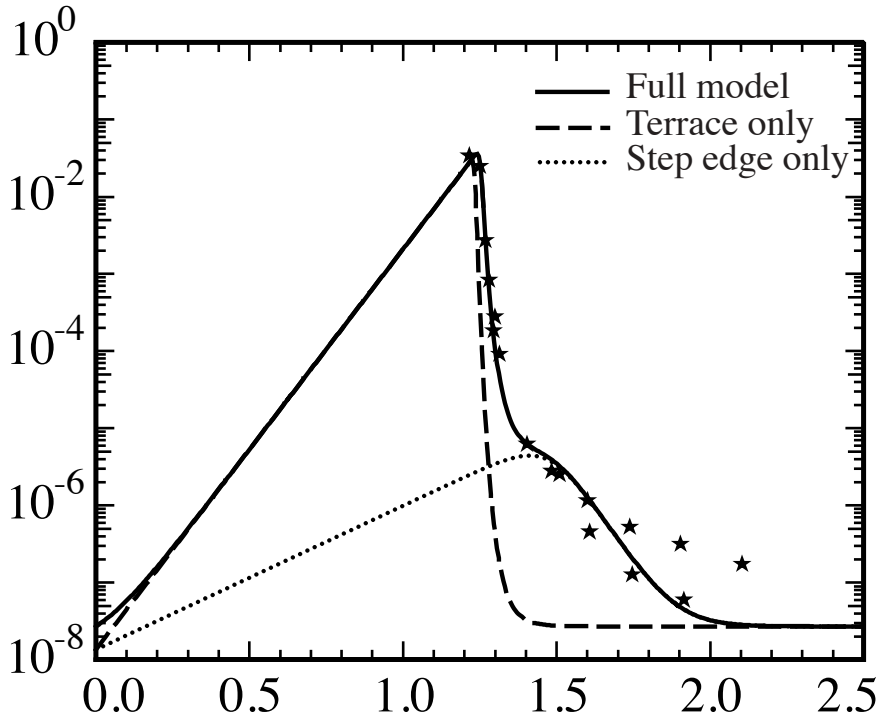

Figure 3. Segregation ratio vs. (T)for $\mathrm{Sb}$ in $\mathrm{Si}(001)$. The solid line represents the fit from our model using the constants and energy barriers given in Table I. The dashed line shows the contribution of the terrace mediated mechanism while the dotted line shows the contribution of the step edge mediated mechanism. Experimental data comes from the literature $[14,15]$ 


\section{SUMMARY}

We have developed a new kinetic model for segregation that can incorporate both step-edge mediated and terrace mediated mechanisms for segregation. Resulting in simple analytic equations, our model successfully accounts for the experimentally observed temperature transition between kinetic and thermodynamic segregation and is also able to describe the anomalous low temperature segregation behavior observed for $\mathrm{Sb}$ in $\mathrm{Si}(001)$. The energies and prefactors used in our fits are consistent with previously reported vales. We attribute the low temperature behavior to the predominance of a lower activation energy step-edge mediated process that enables additional segregation in these temperature regimes. At higher temperatures, the terrace mediate processes dominate the behavior leading to larger amounts of segregation. This research was supported by NSF-DMR-9727369.

\section{REFERENCES}

1. E. Schubert, Delta-Doping of Semiconductors (Cambridge University Press, Cambridge, 1996), p. 604.

2. Semiconductor Lasers II: Materials and Structures, edited by E. Kapon (Academic Press, San Diego, CA, 1999).

3. P. B. Allen, Solid State Comm. 102, 127 (1997).

4. R. F. C. Farrow, IBM J. of Res. and Dev. 42, 43 (1998).

5. H. J. Gossmann and E. F. Schubert, Critical Reviews in Solid State and Materials Sciences 18, 1 (1993).

6. H. J. Gossmann, E. F. Schubert, D. J. Eaglesham, and M. Cerullo, Applied Physics Letters 57, 2440 (1990).

7. M. J. Aziz, J. Appl. Phys. 53, 1158 (1982).

8. J. J. Harris et al., Appl. Phys. A 33, 87 (1984), predates Jorke.

9. H. Jorke, Surf. Sci. 193, 569 (1988).

10. J. Tsao, Materials Fundamentals of Molecular Beam Epitaxy (Academic Press, Boston, 1993), pp. 275-283.

11. D. E. Jesson, S. J. Pennycook, J. M. Baribeau, and D. C. Houghton, Phys. Rev. Lett. 68, 2062 (1992).

12. J. Nutzel and G. Abstreiter, Phys. Rev. B 53, 13551 (1996).

13. L. M. Goldman and M. J. Aziz, J. Mater. Res. 2, 524 (1987).

14. Z. M. Jiang et al., Thin Solid Films 336, 236 (1998).

15. H. Jorke, H. J. Herzog, and H. Kibbel, Fresenius J. Anal. Chem. 341, 176 (1991). 